\title{
Tracking de atividade física em escolares entre 2010 e 2012
}

\author{
Tracking of physical activity in students between 2010 and 2012
}

Renan Camargo Corrêa', Emmanuel Garcia de Toledo e Silva', Antonio Stabelini Neto', Waynne Ferreira de Faria', João Paulo de Farias', Rui Gonçalves Marques Elias', Gessika Castilho dos Santos’

\section{Resumo}

O rastreamento das mudanças na atividade física ao longo do tempo é importante para elaboração de intervenções para a promoção de saúde. Assim, este estudo objetiva analisar o tracking da atividade física (AF) entre 2010 e 2012 em escolares de Jacarezinho-PR. Foram investigados adolescentes do $8^{\circ}$ ano do ensino fundamental que já tinham participado do levantamento de 2010. A amostra foi de 321 indivíduos (47\% meninos). A AF foi avaliada pelo questionário de Baecke nos domínios: escolar, esportivo e lazer. A análise dos dados foi realizada por meio da média e desvio padrão. O teste $t$ pareado foi empregado para comparar a AF entre os momentos e o coeficiente de correlação de Spearman para relacionar os dados obtidos em 2010 e 2012. O domínio escolar apresentou

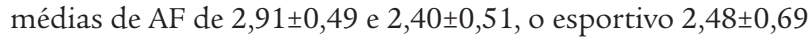

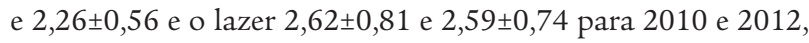
respectivamente. $\mathrm{Na}$ correlação entre os períodos de avaliação, os meninos demostraram valores de correlação significativa entre os valores do índice total $(r=0,302)$, enquanto o gênero feminino apresentou correlação significativa no índice esportivo $(\mathrm{r}=0,344)$, índice de lazer $(\mathrm{r}=0,322)$ e índice total $(\mathrm{r}=0,433)$. Diante desses achados, observa-se que o AF diminui com o avanço da idade e a prática de atividade física demonstrou baixa estabilidade temporal mesmo com o curto período de intervalo entre as avaliações, tendo como resultado o tracking baixo a moderado.

\section{Palavras-chave}

Atividade motora; Tracking; Adolescentes

\begin{abstract}
The tracking of the behavioral changes related to physical activity over time becomes very important for creation of intervention methodologies for health. In this sense, the objective of this study was to analyze the tracking of physical activity index (PAI) between 2010 and 2012 schoolchildren from the city of Jacarezinho-PR. The population consisted of adolescents 8 th grade of elementary school who had already participated in the 2010 collection of data. The sample consisted 321 group of individuals, with 151 boys. The Baecke questionary was used to evaluate the IAF fields: school, sports and leisure time. Data analysis experience was assigned descriptively using mean and standard deviation. The paired t test was used to compare the IAF between moments and the Spearman correlation coefficient to relate data obtained in 2010 and 2012. IAF The field school had $2.91 \pm 0.49$ and $2.40 \pm 0,51$, the sporting $2.48 \pm 0.69$ and $2.26 \pm 0.56$ and $2.62 \pm 0.81$ for leisure and $2.59 \pm$ 0.74 for 2010 and 2012, respectively. The correlation between the evaluation periods, the boys demonstrated a significant correlation between the values the total score $(r=0.302)$, while females showed a significant correlation in the sports index $(r=0.344)$, leisure index $(r=0.322)$ and the total score $(r=0.433)$. At this outcome, it is observed that the IAF decreases with advancing age and physical activity showed low stability even with the short interval between assessments.
\end{abstract}

\section{Keywords}

Motor Activity; Tracking; Teens

\section{Introdução}

Um conjunto de indicadores de saúde e hábitos de vida contribuem para a modificação do perfil de morbimortalidade da população, colocando a inatividade física como prioridade da área de saúde pública, visto que está diretamente relacionada à ocorrência de di-

1 Universidade Estadual do Norte do Paraná, Centro de Ciências da Saúde, Jacarezinho, Paraná, Brasil. versas doenças e agravos não transmissíveis ${ }^{1,2}$.

Evidências apontam que o estilo de vida ativo incorporado na infância e na adolescência pode ser mantido na fase adulta, atuando assim como forma de prevenção e/ou manutenção da saúde e do bem estar $^{3,4}$. Dessa maneira, as recomendações para tal comportamento são amplamente defendidas por diversas entidades nacionais e internacionais ${ }^{5-7}$. Assim, além de descrever a prevalência de atividade física de 
uma população, se torna de suma importância realizar o acompanhamento deste comportamento no que se refere a pratica em domínios distintos ${ }^{8}$.

Estudos de acompanhamento da prática de atividade física em crianças e adolescentes utilizam com frequência o termo tracking para fazer referência as variações deste comportamento com o passar dos anos. Alguns autores ${ }^{9,10}$ tem reportado que 0 tracking da atividade física durante a infância até o final da adolescência é moderado ou fraco. Nesse cenário, ao considerar as categorias de risco do comportamento sedentário, existe uma forte tendência para os adolescentes permanecerem ou reduzirem seus níveis ao longo dos anos ${ }^{3}$. Tendo em vista essa oscilação do comportamento da atividade física, os estudos de tracking devem implicar como forma de diagnóstico do comportamento da população, servindo aos órgãos de saúde como base para traçar estratégias para melhora/manutenção da atividade física ao longo da vida.

Assim, devido a importância do monitoramento da atividade física em escolares e suas possíveis consequências a saúde, o presente estudo teve como objetivo analisar o tracking de atividade física em escolares de Jacarezinho, Paraná, entre os anos de 2010 e 2012.

\section{Métodos}

A população de Jacarezinho-PR tem aproximadamente 40.000 habitantes ${ }^{12}$, sendo que destes 5.242 habitantes estavam matriculados em 2010 entre as $5^{\text {a }}$ série do ensino médio e o $3^{\circ}$ série do ensino médio ano do nos anos finais do ensino fundamental. A população do estudo foi selecionada a partir dos seguintes critérios: a) número total de escolares; b) intervalo de confiança de $95 \%$; c) erro amostral de $5 \%$ e prevalência de $20 \%$, diante dos cálculos foi estimado amostra mínima de 235 escolares, todos os alunos que estavam presentes nos dias das coletas foram convidados a participarem do estudo, a pesquisa foi realizada nos períodos matutino e vespertino, a amostra foi composta por estudantes do ensino fundamental que estavam na $5^{\mathrm{a}}$ série em 2010 e no $7^{\mathrm{a}}$ serie em 2012 . Todas as sete escolas do município participaram do estudo. Dos 510 avaliados, 189 sujeitos foram excluídos por não preencher os dados do questionário corretamente ou não ter participado da primeira avaliação em 2010, restando assim 151 meninos e 170 meninas, totalizando 321 adolescentes.

A AF foi mensurada a partir do questionário proposto por Baecke et al. ${ }^{13}$, o qual contem 16 questões divididas em três sessões: Sessão 1 - Atividade na escola: composta de oito questões, sendo a primeira questão dissertativa e as restantes objetivas dois a oito com um escore de um a cinco pontos. As questões de múltipla escolha são classificadas entre nunca, raramente, algumas vezes, frequentemente e sempre. Nessa sessão os adolescentes foram instruídos a responderem referente ao ambiente escolar; Sessão 2 - Atividades esportivas, programas de exercício físicos e lazer: composta de quatro questões ${ }^{9-12}$, Sessão 3 - Atividade de ocupação do tempo livre: composta de quatro questões ${ }^{13-16}$ com pontuação de um a cinco. $\mathrm{O}$ cálculo para determinação dos índices de atividade na escola, esporte, lazer e total foi realizado conforme as equações desenvolvidas pelo autor ${ }^{13}$, sendo índice de atividade na escola $=\left[\mathrm{Q}_{1}+\left(6-\mathrm{Q}_{2}\right)+\mathrm{Q}_{3}+\mathrm{Q}_{4}+\mathrm{Q}_{5}+\mathrm{Q}_{6}+\mathrm{Q}_{7}+\mathrm{Q}_{8}\right] / 8$, índice esportivo $=$ $\left[\mathrm{Q}_{9}+\mathrm{Q}_{10}+\mathrm{Q}_{11}+\mathrm{Q}_{12}\right] / 4$, e o índice de atividade no lazer $\left[\left(6-\mathrm{Q}_{13}\right)+\mathrm{Q}_{14}+\mathrm{Q}_{15}+\right.$ $\left.\mathrm{Q}_{16}\right] / 4$, já o índice total é a soma dos índices de escolar, índice esportivo e do índice de lazer, este questionário foi traduzido e validado para a língua portuguesa ${ }^{14}$.

Os dados foram analisados utilizando o software SPSS, versão 15.0. A análise dos dados foi atribuída de modo descritivo utilizando média e desvio padrão. Teste t pareado foi empregado para comparar o AF entre os dois momentos avaliados e 
coeficientes de correlação de Spearman para relacionar os dados obtidos em 2010 e 2012, sendo o nível de significância estipulado em $\mathrm{p}<0,05$ para todas as análises.

Este projeto foi aprovado pelo comitê de ética em pesquisa da Universidade Estadual do Norte do Paraná, processo n 060/2012. Os alunos foram avaliados após receberem explicação dos objetivos do estudo e após a devolução do termo de consentimento livre e esclarecido assinado pelos pais ou responsável.

\section{Resultados}

Na tabela 1 observa-se que houve um declínio do índice de AF entre 2010 e 2012 nos escolares. Em relação aos domínios de AF, observa-se que nos meninos houve redução significativa no domínio escolar e total, enquanto as meninas obtiveram diminuição em todos os domínios $(\mathrm{p}<0,05)$.

TABELA 1 - Descrição dos IAF dos escolares de Jacarezinho em 2010 e 2012, estratificado por gênero.

\begin{tabular}{|c|c|c|c|c|}
\hline & 2010 & 2012 & $T$ & $P$ \\
\hline \multicolumn{5}{|c|}{ Índice de AF no domínio Escolar } \\
\hline Masculino & $2,90 \pm 0,53$ & $2,43 \pm 0,52$ & 8,56 & $0,001^{* *}$ \\
\hline Feminino & $2,92 \pm 0,45$ & $2,38 \pm 0,50$ & 11,312 & $0,001^{* *}$ \\
\hline Total & $2,91 \pm 0,49$ & $2,40 \pm 051$ & 14,041 & $0,001^{* *}$ \\
\hline \multicolumn{5}{|c|}{ Índice de AF no domínio Esportivo } \\
\hline Masculino & $2,64 \pm 0,70$ & $2,41 \pm 0,53$ & 1,82 & 0,070 \\
\hline Feminino & $2,33 \pm 0,65$ & $2,13 \pm 0,54$ & 2,057 & $0,041^{*}$ \\
\hline Total & $2,48 \pm 0,69$ & $2,26 \pm 0,56$ & 4,871 & $0,001^{* *}$ \\
\hline \multicolumn{5}{|c|}{ Índice de AF no domínio Lazer } \\
\hline Masculino & $2,69 \pm 0,87$ & $2,78 \pm 0,78$ & $-1,07$ & 0,284 \\
\hline Feminino & $2,56 \pm 0,74$ & $2,42 \pm 0,65$ & 7,740 & $0,001^{* *}$ \\
\hline Total & $2,62 \pm 0,81$ & $2,59 \pm 074$ & 0,643 & 0,521 \\
\hline \multicolumn{5}{|c|}{ Índice de AF Total } \\
\hline Masculino & $8,24 \pm 1,36$ & $7,63 \pm 1,22$ & 4,11 & $0,001^{* *}$ \\
\hline Feminino & $7,82 \pm 1,25$ & $6,93 \pm 1,20$ & 7,740 & $0,001^{* *}$ \\
\hline Total & $8,02 \pm 1,32$ & $7,26 \pm 1,26$ & 9,624 & $0,001^{* *}$ \\
\hline
\end{tabular}

${ }^{*} p<0,05 ;{ }^{* *} p<0,01$.

A tabela 2 apresenta os coeficientes de correlação entre os IAF de 2010 e 2012. Nos meninos, observa-se coeficientes variando entre fraco e muito fraco, sendo que o índice de AF total mostrou correlação significativa entre os momentos (2010 vs 2012). Em relação ao gênero feminino, observa-se coeficientes de correlação de moderado a fraco, com valores significativos para os índices de AF esportivo, lazer e total.

TABELA 2 - Coeficiente de correlação entre os índices de atividade física em escolares entre 2010 e 2012.

\begin{tabular}{|c|c|c|c|c|c|c|c|c|}
\hline & \multicolumn{4}{|c|}{2010} & \multicolumn{4}{|c|}{2012} \\
\hline & \multicolumn{2}{|c|}{ Domínio Escolar } & \multicolumn{2}{|c|}{ Domínio Esportivo } & \multicolumn{2}{|c|}{ Domínio Lazer } & \multicolumn{2}{|c|}{ AF Total } \\
\hline & M & $\mathrm{F}$ & M & $F$ & M & $\mathrm{F}$ & M & $\mathrm{F}$ \\
\hline Domínio Escolar & 0,151 & 0,137 & & & & & & \\
\hline Domínio Esportivo & & & 0,156 & $0,344^{* *}$ & & & & \\
\hline Domínio Lazer & & & & & 0,253 & $0,322^{* *}$ & & \\
\hline AF Total & & & & & & & $0,302^{* *}$ & $0,433^{* *}$ \\
\hline
\end{tabular}

** $p<0,01$. M: Meninos; F: Meninas. 


\section{Discussão}

Os resultados apontam que $\mathrm{AF}$ entre os períodos investigados reduziu significativamente independentemente do gênero. Esses resultados corroboram com os estudos prévios que demonstraram que a atividade física tende a diminuir com o avanço da idade, especialmente durante a adolescência ${ }^{(15-18)}$, inclusive no Brasil ${ }^{19,20}$.

Raudsepp et al ${ }^{21}$ analisaram durante 22 meses o tracking da atividade física em estudantes com idades entre 11 e 12 anos e verificaram uma redução significativa em ambos os gêneros. Da mesma maneira, Pate et al ${ }^{22}$ avaliaram o tracking da atividade física com um intervalo de dois anos em meninas. A cada ano, dependendo da variável investigada, observou-se uma queda de 6 a 13\% do nível de atividade física. Resultado semelhante ao apresentado nesse estudo foi relatado por Knowles et $\mathrm{a}^{23}$ que observou que a $\mathrm{AF}$ reduziu de 3,06 para 2,78 $(\mathrm{p}<0,01)$ após um ano de acompanhamento. Assim, observa-se que a AF tende a diminuir significativamente com o avanço da idade em meninos e meninas durante a adolescência.

Quando o IAF foi estratificado pelos diferentes domínios, o gênero masculino apresentou redução significativa no domínio escolar e no índice total, enquanto os valores para o gênero feminino reduziram significativamente em todos os índices avaliados. Esses resultados estão de acordo com os apresentados por Viana e Andrade ${ }^{24}$, no qual foi observado que quando comparados os gêneros, os meninos mostram uma propensão maior para a aderência da atividade física, uma vez que as meninas geralmente, por questões socioculturais, têm menos incentivo a prática de atividades esportivas. Desta forma, o gênero feminino parece ser uma população mais susceptível as categorias de risco da relacionadas a baixa prática de atividade física.

Correlacionando os domínios do IAF entre os dois momentos, identificou-se entre os meninos uma relação significativa apenas no índice total, enquanto o gênero feminino apresentou significância para os índices de lazer, esportivo e total. Semelhantes resultados foram encontrados por Kjønniksen et $\mathrm{al}^{4}$ que observaram coeficientes de correlação significativos para o gênero masculino $(r=0,21)$ e feminino $(0,23)(\mathrm{p} \geq 0,005)$. Uma possível explicação para a relação significativa entre os momentos e os diferentes domínios da atividade física entre as meninas encontrados no presente estudo, pode ser em virtude de as mesmas terem apresentado baixos índices de atividade física no primeiro momento, havendo assim uma maior "estabilidade" deste comportamento.

Programas de intervenção devem ser discutidos para promover a adesão e manutenção de hábitos saudáveis que influenciam diretamente na saúde e que resultam em melhores perspectivas em termos de qualidade de vida. Nesse sentido, a análise do comportamento da AF na adolescência pode fornecer informações valiosas que são de suma importância para a criação de políticas públicas a fim de mudar esse quadro que abrange proporções nacionais e internacionais ${ }^{18}$. É oportuno ressaltar que este estudo apresenta algumas limitações que merecem ser mencionadas como o período relativamente curto de acompanhamento e o método de mensuração da AF, que mesmo sendo validado para adolescentes ${ }^{14}$, sofre influência do nível de compreensão e interpretação do respondente.

Desta forma, novas pesquisas com maior período de acompanhamento e métodos semelhantes de mensuração da atividade física devem ser conduzidas a fim de melhorar o esclarecimento sobre a tendência da prática de atividade física e as transições comportamentais comuns neste período da vida.

De acordo com os dados dispostos no presente estudo, ao comparar os índices de atividade física entre o intervalo de tempo das avaliações, observa-se que este 
reduziu significativamente independentemente do gênero. Além disso, quando correlacionados os domínios da atividade física entre os períodos, encontrou-se valores de moderada a fraca estabilidade do índice de atividade física.

\section{Agradecimentos}

Agradecemos a Fundação Araucária pela concessão das bolsas de iniciação científica e ao Grupo de Pesquisa em Estilo de Vida, Exercício e Saúde (GPEVES).

\section{Colaboração}

Renan Camargo Corrêa, Emmanuel Garcia de Toledo e Silva e Antonio Stabelini Neto: Concepção, projeto, análise e interpretação dos dados, redação do artigo e revisão crítica relevante do conteúdo intelectual, aprovação final da versão a ser publicada. Waynne Ferreira de Faria, João Paulo de Farias, Rui Gonçalves Marques Elias e Gessika Castilho dos Santos: redação do artigo e revisão crítica relevante do conteúdo intelectual, aprovação final da versão a ser publicada.

\section{Referências}

1. Haskell WL, Lee I-M, Pate RR, Powell KE, Blair SN, Franklin B a, et al. Physical activity and public health: updated recommendation for adults from the American College of Sports Medicine and the American Heart Association. Circulation. 2007 28;116(9):1081-93.

2. Blair SN. Physical inactivity: the biggest public health problem of the 21 st century. Br J Sports Med. $2009 ; 43(1): 1-2$.

3. Azevedo M, Araújo C, Silva M, Hallal P. Tracking of physical activity from adolescence to adulthood: a population-based study. Rev Saude Publica. 2007;41(1):69-75.

4. Kjønniksen L, Torsheim T, Wold B. Tracking of leisure-time physical activity during adolescence and young adulthood: a 10-year longitudinal study. Int J Behav Nutr Phys Act. $2008 ; 69(5): 1-11$.

5. Leitão M, Lazzoli J, Oliveira M, Nóbrega A, Silveira G, Carvalho T, et al. Posicionamento oficial da Sociedade Brasileira de Medicina do Esporte: atividade física e saúde na mulher. Rev Bras Med Esporte. 2000;6(6):215-20.

6. Guedes D, Guedes J. Níveis de prática de atividade física habitual em adolescentes. Rev Bras Med Esporte. 2001;7(6):187-99.

7. Ciolac E, Guimarães G. Exercício físico e síndrome metabólica. Rev Bras Med Esporte. 2004;10(4):319-24.

8. Lopes V, Maia J. Actividade física nas crianças e jovens. Rev Bras Cineantropom Desempenho Hum. 2004;6(1):82-92.

9. Pate R, Trost S, Dowda M. Tracking of physical activity, physical inactivity, and healthrelated physical fitness in rural youth. Pediatr Exerc Sci. 1999;11(4):364-76.

10. Janz KF, Dawson JD, Mahoney LT. Tracking physical fitness and physical activity from childhood to adolescence: the muscatine study. Med Sci Sports Exerc. 2000;32(7):1250-7.

11. Neto AS, Castilho G, Sena J, Campos W. Correlation between physical activity measured by accelerometry and BMI in adolescents. Brazilian J Kinanthropometry Hum Perform. 2013;15(2):174-83.

12. IBGE. Diretoria de Pesquisas - DPE - Coordenação de População e Indicadores Sociais COPIS [online]; Brasil: Instituro Brasileiro de Geografia e Estatistica; 2010. [citado 2014 jun 20]. Disponível em: http://cod.ibge.gov.br/235B8

13. Baecke JA, Burema J, Frijters JE. A short questionnaire for the measurement of habitual physical activity in epidemiological studies. Am J Clin Nutr. 1982;36(5):936-42.

14. Guedes D, Lopes C, Guedes J, Stanganelli L. Reprodutibilidade e validade do questionário Baecke para avaliação da atividade física habitual em adolescentes. Rev Port Ciências do Desporto. 2006;6(3):265-74.

15. Sallis JF. Age-related decline in physical activity: a synthesis of human and animal studies. Med Sci Sports Exerc. 2000;32(9):1598-600. 
16. Bélanger M, Gray-Donald K, O’Loughlin J, Paradis G, Hutcheon J, Maximova K, et al. Participation in organised sports does not slow declines in physical activity during adolescence. Int J Behav Nutr Phys Act. 2009;6(22):1-6.

17. Basterfield L, Adamson AJ, Frary JK, Parkinson KN, Pearce MS, Reilly JJ. Longitudinal study of physical activity and sedentary behavior in children. Pediatrics. 2011;127(1):24-30.

18. Dumith SC, Gigante DP, Domingues MR, Kohl HW. Physical activity change during adolescence: a systematic review and a pooled analysis. Int J Epidemiol. 2011;40(3):685-98.

19. Souza G, Duarte M. Estágios de mudança de comportamento relacionados à atividade física em adolescentes. Rev Bras Med Esporte. 2005;11(2):104-8.

20. Farias Júnior J, Nahas M, Barros M, Loch M, Oliveira E, De Bem M, et al. Comportamentos de risco à saúde em adolescentes no Sul do Brasil: prevalência e fatores associados. Rev Panam Salud Publica. 2009;25(4):344-52.

21. Raudsepp L, Neissaar I, Kull M. Longitudinal stability of sedentary behaviors and physical activity during early adolescence. Pediatr Exerc Sci. 2008;20(3):251-62.

22. Pate RR, Stevens J, Webber L, Dowda M, Murray DM, Young D. Age-related change in physical activity in adolescent girls. J Adolesc Heal. 2009;44(3):275-82.

23. Knowles A-M, Niven AG, Fawkner SG, Henretty JM. A longitudinal examination of the influence of maturation on physical self-perceptions and the relationship with physical activity in early adolescent girls. J Adolesc. 2009;32(3):555-66.

24. Viana M S, Andrade A. Estágios de mudança de comportamento relacionados ao exercício físico em adolescentes. Rev Bras Cineantropometria e Desempenho Hum. 2010;12(5):367-74.

\section{ENDEREÇO PARA}

CORRESPONDÊNCIA

RENAN CAMARGO CORREA

renan_edf91@hotmail.com
Universidade Estadual do Norte do Paraná-

Centro de Ciências da Saúde - Curso de

Educação Física, Grupo de Pesquisa em Estilo de

Vida, Exercício e Saúde. Alameda Padre Magno,

841 - Nova Alcântara, Jacarezinho - PR, 86400-

000. Fone/cel: 4399122818
RECEBIDO 26/08/2014

REVISADO 05/01/2016

APROVADO 08/01/2016 